\title{
On the dynamics of classical particle with spin
}

\author{
Natalia Kudryashova* \\ Department of Mathematics, University College London, \\ Gower Street, London, WC1E 6BT, United Kingdom \\ Yuri N. Obukhovt \\ Department of Mathematics and Institute of Origins, \\ University College London, Gower Street, \\ London, WC1E 6BT, United Kingdom
}

\begin{abstract}
The complete explicitly covariant 4-dimensional description of the dynamics of a free classical particle with spin within the framework of the special relativity theory is presented. The key point of our approach is the the introduction of the new vector field which enables to define the analogues of the mean spin and position variables. The supplementary conditions are discussed and it is demonstrated that the Frenkel condition unambiguously determines the dynamics of a spinning particle.
\end{abstract}

PACS numbers: 04.50.+h; 04.20.Jb; 03.50.Kk

*Electronic address: n.kudryashova@ucl.ac.uk

†Electronic address: obukhov@math.ucl.ac.uk 


\section{INTRODUCTION}

The models of classical spinning matter go back to 1926 when, shortly after Uhlenbeck and Goudsmit [1] have introduced the notion of intrinsic angular momentum in quantum mechanics, J. Frenkel [2] developed the theory of a classical electron with spin moving in electromagnetic field. Since that time, the dynamics of classical spinning matter (particles and continuous media) in special and general relativity was studied intensively. For the overview see [3].

Although the true understanding of spin as an essentially quantum property of matter is achieved only in quantum theory, the classical models nevertheless turn out to be rich and consistent to an extent which justifies their applications for the analysis of the experiments with the high energy beams of polarised particles and with the polarised targets. That direction was brought to life by the work of Bargmann, Michel, and Telegdi [4] who established that the rigorous quantum theory practically exactly reproduces the Frenkel model when the spin variable is defined as a mean value of the quantum spin operator over a quasi-classical wave packet state.

Considerable attention was paid to the study of dynamics of spinning particles in the gravitational field, see for example [5-7], a historic account and the list of the references can be found in [8]. Among other issues, the choice of the so-called supplementary condition was a subject of a long discussion. The two main options are Frenkel's and Tulczyjew's conditions. Comparing these choices, one finds the statements in the literature about the "ambiguity" allowed for particle's trajectory under the Frenkel condition [7, 13]. We will address this issue here.

In this paper we reconsider the dynamics of a classical particle with spin within the framework of the special relativity theory. Although much of the work was done previously,

see, e.g., [9, 10], the problem was usually considered within the 3-dimensional framework (often using special reference systems). Our aim is to describe the explicitly covariant 4-dimensional general picture of a spinning particle dynamics. As a result, we will find certain new features, especially by making a careful comparison of Frenkel's and Tulczyjew's supplementary conditions. We demonstrate that the use of the Frenkel condition yields a trajectory uniquely determined by the initial values of the momentum, spin and particle's position. 


\section{CLASSICAL MODEL OF PARTICLE WITH SPIN}

We will confine our attention to a point particle with spin described by the FrenkelWeyssenhoff theory [2, 9, 10]. In this classical model, a spinning particle is described as a physical point which position in the Minkowski spacetime is given by the four coordinates,

$$
X^{\mu}=X^{\mu}(\tau)
$$

and which is characterised by the two gravitational "charges", the 4-momentum vector and the spin tensor:

$$
\begin{aligned}
P_{\alpha} & =P_{\alpha}(X(\tau)), \\
S_{\alpha \beta} & =S_{\alpha \beta}(X(\tau)) .
\end{aligned}
$$

The parameter $\tau$ can be chosen as an arbitrary Lorentz-invariant real variable. Without loosing generality, we will assign $\tau=0$ to the initial position of a particle. As usual, we also assume that $\tau$ is equal to the proper time along the world line of a particle, so that the 4-velocity,

$$
U^{\mu}=\frac{d X^{\mu}}{d \tau}
$$

is normalised by the condition

$$
U^{\mu} U_{\mu}=1
$$

Finally, we assume the supplementary condition

$$
S_{\alpha \beta}\left(\hat{\alpha} U^{\beta}+\hat{\beta} P^{\beta}\right)=0
$$

with the constant parameters $\hat{\alpha}$ and $\hat{\beta}$. This general condition includes as limiting cases a so-called Frenkel [2] (sometimes also called Pirani [12]) and Tulczyjew conditions [13], with $\hat{\beta}=0$ and $\hat{\alpha}=0$, respectively. The Frenkel condition corresponds to the spin of pure "magnetic" nature, and physically means that in the rest frame of a particle the spin has only three spatial components. Under the Tulczyjew's condition, the trajectory of an extended test body is determined by the position of the centre of mass of the body.

Dynamics of a free particle is governed by the standard conservation laws of momentum and of the total angular momentum,

$$
\begin{aligned}
\frac{d}{d \tau} P_{\alpha} & =0 \\
\frac{d}{d \tau}\left(X_{\alpha} P_{\beta}-X_{\beta} P_{\alpha}+S_{\alpha \beta}\right) & =0 .
\end{aligned}
$$


Although this system looks rather simple, its complete integration is nontrivial. As a first step, denoting the initial values of the particle position, momentum, and spin by $\stackrel{(0)}{X}^{\mu}, \stackrel{(0)}{P} \alpha, \stackrel{(0)}{S}_{\alpha \beta}$, respectively, we find from the first integrals from (2.7) $-(2.8)$ :

$$
\begin{aligned}
P_{\alpha} & =\stackrel{(0)}{P} \alpha, \\
X_{\alpha} P_{\beta}-X_{\beta} P_{\alpha}+S_{\alpha \beta} & =J_{\alpha \beta},
\end{aligned}
$$

with constant tensor

$$
J_{\alpha \beta}:=\stackrel{(0)}{X}_{\alpha} \stackrel{(0)}{P}_{\beta}-\stackrel{(0)}{X}_{\beta} \stackrel{(0)}{P}_{\alpha}+\stackrel{(0)}{S}_{\alpha \beta} .
$$

For $(4+4+4+6)=18$ unknown variables $X^{\mu}, U^{\mu}, P_{\alpha}, S_{\alpha \beta}$, we have exactly $(4+$ $1+3+4+6)=18$ differential and algebraic equations, (2.4)-(2.8). Or, by substituting (2.4) into (2.5) and (2.6), we have a system of 14 differential-algebraic equations for the 14 variables $X^{\mu}, P_{\alpha}, S_{\alpha \beta}$. Accordingly, we expect that all these dynamical variables are uniquely determined by their initial values.

Combining (2.8) and (2.4), we find:

$$
\dot{S}_{\alpha \beta}=-U_{\alpha} P_{\beta}+U_{\beta} P_{\alpha}
$$

Hereafter the proper time derivative will be denoted by the dot, $\frac{d}{d \tau}=\left({ }^{\circ}\right)$. The system (2.7) and (2.12) was generalised to the case of the curved spacetime by Mathisson and Papapetrou [11] (then the curvature-dependent forces appear on the right-hand side). Contracting (2.12) with $U^{\beta}$, we get

$$
P_{\alpha}=m_{0} U_{\alpha}+U^{\beta} \dot{S}_{\alpha \beta}
$$

where we denote

$$
m_{0}:=U^{\alpha} P_{\alpha} .
$$

It is easy to see that for the Frenkel and the Tulczyjew conditions $m_{0}$ is constant along the world line [contract (2.13) with $\dot{U}^{\alpha}$ and use (2.5) and (2.6) to obtain $\dot{U}^{\alpha} P_{\alpha}=\dot{m}_{0}=0$ ]. This integration constant is naturally interpreted as the rest mass of the particle.

Substituting (2.13) back to (2.12), one can rewrite the equation of motion of spin in the form:

$$
\dot{S}_{\alpha \beta}-U_{\alpha} U^{\gamma} \dot{S}_{\gamma \beta}-U_{\beta} U^{\gamma} \dot{S}_{\alpha \gamma}=0
$$




\section{NEW VECTOR VARIABLE}

Let us contract (2.12) with $P^{\beta}$. The result reads:

$$
\dot{S}_{\alpha \beta} P^{\beta}=-U_{\alpha} P^{2}+m_{0} P_{\alpha}
$$

Assuming that the square of the 4-momentum, $P^{2}:=P_{\alpha} P^{\alpha}$, is non-zero, it is convenient to introduce a new variable

$$
Q_{\alpha}:=\frac{1}{P^{2}} P^{\beta} S_{\alpha \beta},
$$

From the normalisation condition (2.5) for the velocity, we find

$$
1=U^{\alpha} U_{\alpha}=\left(\frac{m_{0}}{P^{2}}\right)^{2} P^{\alpha} P_{\alpha}+\dot{Q}^{\alpha} \dot{Q}_{\alpha}
$$

The equation (3.16) takes the form

$$
U_{\alpha}+\dot{Q}_{\alpha}=\frac{m_{0} P_{\alpha}}{P^{2}}
$$

Recalling (2.4), we can easily integrate the last equation to find:

$$
X_{\alpha}+Q_{\alpha}=\frac{m_{0} P_{\alpha}}{P^{2}} \tau+\stackrel{(0)}{X}_{\alpha}+\stackrel{(0)}{Q_{\alpha}}
$$

From the condition (2.6) it follows that the new variable is orthogonal to the 4-velocity,

$$
Q_{\alpha} U^{\alpha}=0
$$

Hence the vector $Q_{\alpha}$ is spacelike. On the other hand, by construction, $Q_{\alpha}$ is orthogonal also to the 4-momentum,

$$
Q_{\alpha} P^{\alpha}=0
$$

Finally, contracting (3.19) with $Q^{\alpha}$ and using the condition (2.6) together with (3.22), we obtain

$$
Q_{\alpha} \dot{Q}^{\alpha}=0
$$

Substituting (3.20) into (2.8) we find a new representation of the spin tensor:

$$
S_{\alpha \beta}=\mu_{\alpha \beta}+Q_{\alpha} P_{\beta}-Q_{\beta} P_{\alpha},
$$

where

$$
\mu_{\alpha \beta}=J_{\alpha \beta}-\left(\stackrel{(0)}{X}_{\alpha}+\stackrel{(0)}{Q}_{\alpha}\right) P_{\beta}+\left(\stackrel{(0)}{X}_{\beta}+\stackrel{(0)}{Q}_{\beta}\right) P_{\alpha}
$$


is a constant tensor. Comparing with (2.11), one can write its components explicitly in terms of the initial values of spin and momentum:

$$
\mu_{\alpha \beta}=\stackrel{(0)}{S}_{\alpha \beta}-\frac{1}{P^{2}} P_{\alpha} P^{\gamma} \stackrel{(0)}{S}_{\gamma \beta}-\frac{1}{P^{2}} P_{\beta} P^{\gamma} \stackrel{(0)}{S}_{\alpha \gamma}
$$

In other words, $\mu_{\alpha \beta}$ describes the projection of spin on the total momentum $P_{\alpha}$ at the initial moment. If, initially, spin is parallel to the momentum (i.e., the orthogonal projection vanishes, $\left.P^{\gamma} \stackrel{(0)}{S}_{\alpha \gamma}=0\right), \mu_{\alpha \beta}$ reduces to the tensor of spin.

Thus, as soon as the time dependence of the variable $Q_{\alpha}$ is known, the dynamics of the particle (its position and spin as the functions of the proper time) is completely described by (3.20) and (3.24). It is worthwhile to stress that $Q_{\alpha}$ is a composite variable and hence its initial values are not arbitrary, they are fixed by the initial values of momentum and spin.

\section{PHYSICAL SOLUTIONS}

Now, let us find $Q_{\alpha}(\tau)$ explicitly. From (3.26) we discover another orthogonality relation

$$
\mu_{\alpha \beta} P^{\beta}=0
$$

which is crucial to obtain the evolution equation for $Q_{\alpha}$ alone. Indeed, taking $U_{\alpha}$ from (3.19), substituting it into the condition (2.6), and making use of the definition (3.17) and of the relations (3.24) and (4.27), one finds:

$$
\mu_{\alpha \beta} \dot{Q}^{\beta}=\left(\frac{\hat{\beta}}{\hat{\alpha}} P^{2}+m_{0}\right) Q_{\alpha} .
$$

In the course of this derivation we also used (3.22) and (3.23). We assume that $\hat{\alpha} \neq 0$, the case of the vanishing $\hat{\alpha}$ will be analysed separately.

Denote the effective mass:

$$
\tilde{m}_{0}:=\frac{\hat{\beta}}{\hat{\alpha}} P^{2}+m_{0} .
$$

Then, the equation (4.28) can be re-written as:

$$
\mu_{\alpha \beta} \dot{Q}^{\beta}-\tilde{m}_{0} Q_{\alpha}=0
$$


The system of linear differential equations (4.30) is straightforwardly integrated. The standard ansatz $Q_{\alpha}=q_{\alpha} e^{-i \omega \tau}$ leads to the algebraic system for the amplitudes $q_{\alpha}$ which has nontrivial solutions when the characteristic determinant is zero,

$$
\operatorname{det}\left|\tilde{m}_{0} g_{\alpha \beta}+i \omega \mu_{\alpha \beta}\right|=0
$$

The computation of the determinant yields

$$
\tilde{m}_{0}^{2}-\omega^{2} \frac{1}{2} \mu_{\alpha \beta} \mu^{\alpha \beta}=0
$$

because $\varepsilon^{\alpha \beta \mu \nu} \mu_{\alpha \beta} \mu_{\mu \nu}=0$ in view of (4.27).

The nontrivial solutions arise when $\mu_{\alpha \beta} \mu^{\alpha \beta} \neq 0$. In particular, for $\mu_{\alpha \beta} \mu^{\alpha \beta}>0$ the general solution of (4.30) is an oscillatory motion

$$
Q_{\alpha}(\tau)=\nu_{\alpha} \cos (\omega \tau)+\lambda_{\alpha} \sin (\omega \tau)
$$

with the frequency

$$
\omega=\frac{\tilde{m}_{0}}{\sqrt{\frac{1}{2} \mu_{\alpha \beta} \mu^{\alpha \beta}}} .
$$

Here the constant coefficients $\nu_{\alpha}, \lambda_{\alpha}$ are determined by the initial values of the variables. Explicitly, we find

$$
\nu_{\alpha}=\stackrel{(0)}{Q}_{\alpha}=\frac{1}{P^{2}} P^{\beta} \stackrel{(0)}{S}_{\alpha \beta},
$$

and $\lambda_{\alpha}$ is constructed below in Sec. 5. If $\hat{\alpha}$ and $\hat{\beta}$ are such that $\hat{\alpha} U^{\beta}+\hat{\beta} P^{\beta}=0$, i.e. $\tilde{m}_{0}=0$, the frequency of vibrations $\omega$ is zero, so that the corresponding trajectory is a straight line. Also, when $\mu_{\alpha \beta}=0$, the equation (4.30) yields $Q_{\alpha}=0$, and as it follows from (3.20), the particle moves along the straight line:

$$
X_{\alpha}=\frac{\tilde{m}_{0} P_{\alpha}}{P^{2}} \tau+\stackrel{(0)}{X}
$$

In particular, this also means that $\nu_{\alpha}=0$, and $\mu_{\alpha \beta}=\stackrel{(0)}{S}_{\alpha \beta}=0$, i.e. it is a spinless case.

Let us assume that $\mu_{\alpha \beta} \neq 0$. Then (4.27) is equivalent to the existence of a 4 -vector

$$
\mu^{\alpha}:=\frac{1}{2} \varepsilon^{\alpha \beta \rho \sigma} P_{\beta} \mu_{\rho \sigma}
$$

which is orthogonal to the 4-momentum,

$$
\mu^{\alpha} P_{\alpha}=0
$$


The inverse of (4.37) reads

$$
\mu_{\alpha \beta}=\frac{1}{P^{2}} \varepsilon_{\alpha \beta \mu \nu} P^{\mu} \mu^{\nu} .
$$

Substituting this into (4.30), one finds

$$
\frac{1}{P^{2}} \varepsilon_{\alpha \beta \mu \nu} P^{\mu} \mu^{\nu} \dot{Q}^{\beta}-\tilde{m}_{0} Q_{\alpha}=0,
$$

which immediately yields a new orthogonality relation,

$$
\mu^{\alpha} Q_{\alpha}=0 .
$$

\section{PROPERTIES OF OSCILLATORY SOLUTIONS}

Summarising the above results, we observe that the vectors $\left\{P^{\alpha}, \mu^{\alpha}, Q^{\alpha}\right\}$ comprise a triplet of the mutually orthogonal vectors at any moment of proper time $\tau$. More precisely, the pair of constant (and mutually orthogonal, see (4.38)) vectors $P^{\alpha}, \mu^{\alpha}$ define a 2-dimensional plane. In view of (3.22) and (4.41), the variable $Q^{\alpha}$ always lies in the spacelike 2-dimensional plane which is orthogonal to the first plane.

We can finalise the integration of (4.30) as follows. Inserting (4.33) in (4.40), one finds the two relations between the four constant vectors:

$$
\begin{aligned}
\lambda_{\alpha} & =-\frac{\omega}{\tilde{m}_{0} P^{2}} \varepsilon_{\alpha \beta \mu \nu} \nu^{\beta} P^{\mu} \mu^{\nu}, \\
\nu_{\alpha} & =\frac{\omega}{\tilde{m}_{0} P^{2}} \varepsilon_{\alpha \beta \mu \nu} \lambda^{\beta} P^{\mu} \mu^{\nu} .
\end{aligned}
$$

This shows that the constant vectors $\nu_{\alpha}$ and $\lambda_{\alpha}$ are orthogonal to each other,

$$
\nu_{\alpha} \lambda^{\alpha}=0
$$

and to $P^{\alpha}, \mu^{\alpha}$ as well,

$$
\nu_{\alpha} P^{\alpha}=\nu_{\alpha} \mu^{\alpha}=\lambda_{\alpha} P^{\alpha}=\lambda_{\alpha} \mu^{\alpha}=0 .
$$

Substituting (5.42) into (5.43), we find:

$$
\nu_{\alpha}=-\left(\frac{\omega}{\tilde{m}_{0} P^{2}}\right)^{2} \varepsilon_{\alpha \beta \mu \nu} \varepsilon^{\beta \gamma \rho \sigma} \nu_{\gamma} P_{\rho} \mu_{\sigma} P^{\mu} \mu^{\nu}=-\left(\frac{\omega}{\tilde{m}_{0}}\right)^{2} \frac{\mu_{\beta} \mu^{\beta}}{P^{2}} \nu_{\alpha},
$$

where we repeatedly used the orthogonality relations (5.45). As one can straightforwardly verify from (4.37) and (4.39),

$$
\frac{1}{2} \mu_{\alpha \beta} \mu^{\alpha \beta}=-\frac{\mu_{\alpha} \mu^{\alpha}}{P^{2}}
$$


and hence (5.46) is identically satisfied when the frequency $\omega$ is given by (4.34).

Hence, provided the initial conditions for the momentum $\stackrel{(0)}{P}_{\alpha}$ and spin $\stackrel{(0)}{S}_{\alpha \beta}$ are given, one can construct a constant spacetime frame represented by the four vectors:

$$
P^{\alpha}, \quad \mu^{\alpha}, \quad \nu^{\alpha}, \quad \lambda^{\alpha},
$$

as shown in (4.37), (3.17) and (5.42). The four vectors (5.48) are all orthogonal to each other; $P^{\alpha}$ is timelike (i.e., $P^{2}=P_{\alpha} P^{\alpha}>0$ ), whereas the three other are spacelike (thus having negative length square). One can show that the lengths of the three spacelike vectors are proportional to each other. Indeed, squaring (5.42), we find

$$
\lambda_{\alpha} \lambda^{\alpha}=\nu_{\alpha} \nu^{\alpha}
$$

Then from (4.33) we immediately obtain:

$$
\dot{Q}_{\alpha} \dot{Q}^{\alpha}=\omega^{2} Q_{\alpha} Q^{\alpha}
$$

and then using (3.18),

$$
1=U_{\alpha} U^{\alpha}=\frac{m_{0}^{2}}{P^{2}}+\omega^{2} Q_{\alpha} Q^{\alpha} .
$$

From this we find finally (insert (4.34) and (5.47)):

$$
\nu_{\alpha} \nu^{\alpha}=\frac{\mu^{\alpha} \mu_{\alpha}}{P^{2}}\left(\frac{m_{0}^{2}}{P^{2}\left(\frac{\hat{\beta}}{\hat{\alpha}} P^{2}+m_{0}\right)^{2}}-\frac{1}{\left(\frac{\hat{\beta}}{\hat{\alpha}} P^{2}+m_{0}\right)^{2}}\right)
$$

This shows, since $\nu_{\alpha}$ is spacelike, that one must have

$$
P^{2}<m_{0}^{2}
$$

The dynamics of spin is given by (3.24). In order to get a further insight (motivated by the quantum mechanical nature of spin, [16]), one usually introduces the 4-vector of spin by $S^{\alpha}=\frac{1}{2} \varepsilon^{\alpha \beta \rho \sigma} U_{\beta} S_{\rho \sigma}$. Then by using the Mathisson-Papapetrou equations of motion and the results obtained above, we straightforwardly verify that the spin vector precesses as

$$
\dot{S}^{\alpha}=\Omega_{\beta}^{\alpha} S^{\beta}
$$

The precession angular velocity is $\Omega_{\alpha \beta}=-\omega \hat{\mu}_{\alpha \beta}$, where $\hat{\mu}_{\alpha \beta}=\mu_{\alpha \beta} / \sqrt{\frac{1}{2} \mu_{\rho \sigma} \mu^{\rho \sigma}}$ is the unit tensor giving the direction of the precession axis. 


\section{SPECIAL CASES}

Let us summarise the results on the dynamics of a free particle with spin. There are several qualitatively different cases which arise for the different limits of the supplementary condition and for the different initial conditions.

\section{A. Tulczyjew condition}

When $\hat{\alpha}=0$ the condition (2.6) becomes the Tulczyjew's condition:

$$
S_{\alpha \beta} P^{\beta}=0
$$

Consequently, the $Q_{\alpha}$ as defined in the (3.17) vanishes at any $\tau$, meaning that the trajectories are straight lines described by

$$
X^{\alpha}=\frac{P^{\alpha}}{P^{2}} \tau+\stackrel{(0)}{X^{\alpha}},
$$

together with constant momentum and spin

$$
P_{\alpha}=\stackrel{(0)}{P}_{\alpha}, \quad S_{\alpha \beta}=\stackrel{(0)}{S}_{\alpha \beta} .
$$

\section{B. Frenkel condition}

When $\hat{\beta}=0$, the Frenkel condition, is recovered:

$$
S_{\alpha \beta} U^{\beta}=0
$$

Under the Frenkel condition, $m_{0}$ is constant along the world line and the oscillation frequency is

$$
\omega=\frac{m_{0}}{\sqrt{\frac{1}{2} \mu_{\alpha \beta} \mu^{\alpha \beta}}} .
$$

One can establish several other useful relations between spin and momentum. For example, directly from (3.24) one sees:

$$
\frac{1}{2} S_{\alpha \beta} S^{\alpha \beta}=\frac{1}{2} \mu_{\alpha \beta} \mu^{\alpha \beta}+P^{2} Q_{\alpha} Q^{\alpha}
$$

and thus using (5.52), we find

$$
\frac{1}{2} S_{\alpha \beta} S^{\alpha \beta}=\frac{1}{2} \mu_{\alpha \beta} \mu^{\alpha \beta} \frac{P^{2}}{m_{0}^{2}}
$$


This suggests another form for the frequency:

$$
\omega=\frac{|P|}{\sqrt{\frac{1}{2} S_{\alpha \beta} S^{\alpha \beta}}},
$$

where $|P|=\sqrt{P_{\alpha} P^{\alpha}}$. Interesting to note that one can rewrite the above equation in the form

$$
|P|=|S| \omega
$$

which with $|S|=\sqrt{\frac{1}{2} S_{\alpha \beta} S^{\alpha \beta}}$ resembles the well-known wave-particle relation $(E=h \nu)$ if one estimates $|S|=\hbar / 2$ and replaces $\omega=2 \pi \nu$. With these identifications, $\omega$ coincides with the Zitterbewegung frequency derived in the quantum theory.

\section{Straight line motion}

We have seen that the cases of spinless particles, as well as the conditions $\hat{\alpha}=0$ and $\hat{\alpha} U^{\beta}+\hat{\beta} P^{\beta}=0$ correspond to the straight line motion.

Furthermore, one can show that the particle with spin moves monotonously along a straight line if and only if, at an initial moment $\tau=0$

$$
\stackrel{(0)}{S} \stackrel{(0)}{\alpha \beta}^{\beta}=0 .
$$

Then the particle's position is described by (4.36), while the spin is constant,

$$
S_{\alpha \beta}=\mu_{\alpha \beta}
$$

The proof is as follows. The initial condition (6.64) is equivalent to $\nu_{\alpha}=0$, so when this is satisfied, we find from (4.33), (5.42) that $Q_{\alpha}=0$ for any $\tau$. Hence, (3.20) reduces to (4.36) and (3.24) to (6.65). On the other hand, suppose the particle moves along a straight line $X_{\alpha}=C_{\alpha} \tau+\stackrel{(0)}{X}$ with some constant vector $C_{\alpha}$. Then $U_{\alpha}=C_{\alpha}$, and thus $U^{\beta} \dot{S}_{\alpha \beta}=\frac{d}{d \tau}\left(U^{\beta} S_{\alpha \beta}\right)=0$. Hence the equation (2.13) reduces to $P_{\alpha}=m_{0} U_{\alpha}$ which gives the constant vector $C_{\alpha}=P_{\alpha} / m_{0}$, and we again recover (4.36). At the same time, the Frenkel condition (2.6) then yields (6.64).

\section{Classical Zitterbewegung}

When, at an initial moment $\tau=0$,

$$
\stackrel{(0)}{S} \underset{\alpha \beta}{P^{\beta}} \neq 0
$$


the particle's motion is described by (3.20) and (3.24), where the variable $Q_{\alpha}$ oscillates according to (4.33) with the frequency (4.34). This picture is a classical counterpart of the Zitterbewegung of a Dirac particle in relativistic quantum mechanics. There is a natural global orthogonal frame (5.48) in which this motion is described in a most clear way.

It seems worthwhile to note that the explicit 4-dimensional picture makes more transparent the details of the classical oscillatory motion. In particular, contrary to the statements which can be encountered in the 3-dimensional analyses (see, e.g., [9, 10]), the dynamics of spinning particle is not a mere superposition of a motion along a line with a rotation around the direction of momentum or around the direction of spin. More precisely, we have learned that the particle's rotary motion is confined to the plane $\left(\nu_{\alpha}, \lambda_{\alpha}\right)$ orthogonal to both the momentum and spin.

\section{NON-PHYSICAL SOLUTIONS}

One can verify that the relation (6.61) holds true in general, and not only for the above case when $\mu_{\alpha \beta} \mu^{\alpha \beta}>0$ (then this relation confirms that the momentum in timelike).

Correspondingly, we read from (6.61) that assuming the unphysical spacelike momentum, i.e., $P^{2}<0$, we automatically find that $\mu_{\alpha \beta} \mu^{\alpha \beta}<0$. Returning now to the linear system (4.30), we see that instead of the oscillatory solutions (4.33) we find the hyperbolic motion:

$$
Q_{\alpha}(\tau)=\nu_{\alpha} \cosh (\bar{\omega} \tau)+\lambda_{\alpha} \sinh (\bar{\omega} \tau)
$$

with the "acceleration" parameter

$$
\bar{\omega}=\frac{\tilde{m}_{0}}{\sqrt{-\frac{1}{2} \mu_{\alpha \beta} \mu^{\alpha \beta}}} .
$$

One can immediately see that the constant vectors $\nu_{\alpha}$ and $\lambda_{\alpha}$ satisfy the relations

$$
\nu_{\alpha} \nu^{\alpha}=-\lambda_{\alpha} \lambda^{\alpha}, \quad \nu_{\alpha} \lambda^{\alpha}=0
$$

Consequently, since the variable $Q^{\alpha}$ is always spacelike, we discover that the vector $\nu_{\alpha}$ is also spacelike, whereas $\lambda_{\alpha}$ is timelike. Recalling that (5.47) in all cases describes the vector $\mu_{\alpha}$ as spacelike, we thus again end with the global vierbein (5.48), where however the role of the timelike leg is played by the vector $\lambda_{\alpha}$ instead of the momentum. 
The existence of such a solution was mentioned recently in [14]. It is clear that the self-accelerated solutions of the type (7.67) (recall that the particle is not affected by any external forces) are non-physical and one should exclude them. This imposes a transparent constraint on the possible values of the momentum: $P^{2}>0$.

\section{CONCLUSION}

In this paper we have presented a complete explicitly covariant 4-dimensional description of the dynamics of a free classical particle with spin in the framework of the special relativity theory. The crucial step in the analysis of the problem was provided by the introduction of the new vector variable $Q^{\alpha}$. From (3.24) and (3.20), one can notice a certain analogy with the definition of the mean spin and mean position operators in relativistic quantum mechanics [15]. Finally, we demonstrated that, contrary to the earlier statements [7, 13], the dynamics of a spinning particle (both the motion of spin and the trajectory) under the Frenkel condition is uniquely determined by the initial conditions, i.e., by the values of $\stackrel{(0)}{X}^{\alpha}, \stackrel{(0)}{P}_{\alpha}, \stackrel{(0)}{S}_{\alpha \beta}$. This important property is valid also for the curved spacetimes, as we will show elsewhere.

[1] G.E. Uhlenbeck and S. Goudsmit, Ersetzung der Hypothese vom unmechanischen Zwang durch eine Forderung bezüglich des inneren Verhaltens jedes einzelnen Elektrons, Naturwiss. 13 (1925) 953.

[2] J. Frenkel, Die Elektrodynamik des rotierenden Elektrons, Zeits. Phys. 37 (1926) 243-262.

[3] H.C. Corben, Classical and quantum theories of spinning particles (Holden-Day, Inc: San Francisco, 1968); I.M. Ternov, Introduction to physics of spin of elementary particles (Moscow State University Publ. House: Moscow, 1997), in Russian.

[4] V. Bargmann, L. Michel, and V.L. Telegdi, Precession of the polarization of particles moving in a homogeneous electromagnetic field, Phys. Rev. Lett. 2 (1959) 435-436.

[5] B. Mashhoon, Particle with spin in a gravitational field, J. Math. Phys. 12 (1971) 1075-1077.

[6] M. Mohseni, Spinning particles in gravitational wave spacetime, Phys. Lett. A 301 (2002) 382-388; M. Mohseni, Motion of pole-dipole and quadrupole particles in non-minimally coupled 
theories of gravity, Phys. Rev. D 81 (2010) 124039 (6 pages).

[7] O. Semerák, Spinning test particles in a Kerr field - I, Monthly Notices Roy. Astron. Soc. 308 (1999) 863-875; K. Kyrian and O. Semerák, Spinning test particles in a Kerr field - II, Monthly Notices Roy. Astron. Soc. 382 (2007) 1922-1932.

[8] D. Puetzfeld and Yu.N. Obukhov, Probing non-Riemannian spacetime geometry, Phys. Lett. A 372 (2008) 6711-6716; D. Puetzfeld and Yu.N. Obukhov, Propagation equations for deformable test bodies with microstructure in extended theories of gravity, Phys. Rev. D 76 (2007) 084025 (20 pages).

[9] J. Weyssenhoff and A. Raabe, Relativistic dynamics of spin-fluids and spin-particles, Acta Phys. Pol. 9 (1947) 7-18.

[10] H.C. Corben, Spin in classical and quantum theory, Phys. Rev. 121 (1961) 1833-1839; H.C. Corben, Spin precession in classical relativistic mechanics, Nuovo Cim. 20 (1961) 527-541.

[11] M. Mathisson, Neue Mechanik materieller Systeme, Acta Phys. Pol. 6 (1937) 163-200; A. Papapetrou, Spinning test-particles in general relativity. I, Proc. Roy. Soc. London A209 (1951) $248-258$.

[12] F.A.E. Pirani, On the physical significance of the Riemann tensor, Acta Phys. Polon. 15 (1956) 389-405.

[13] W.Tulczyjew, Motion of multipole particles in general relativity theory, Acta Phys. Pol. 18 (1959) 393-409.

[14] I.B. Khriplovich, Spinning relativistic particles in external fields, in: "Gyros, clocks, interferometers...: Testing relativistic gravity in space" (Springer: Berlin, 2001) C. Lämmerzahl, C.W.F. Everitt, and F.W. Hehl (Eds.), Lect. Notes Phys. 562 (2001) 109-128.

[15] T.D. Newton and E.P. Wigner, Localized states for elementary systems, Rev. Mod. Phys. 21 (1949) 400-406; L.L. Foldy and S.A. Wouthuysen, On the Dirac theory of spin 1/2 particles and its non-relativistic limit, Phys. Rev. 78 (1950) 29-36; J. Hilgevoord and S.A. Wouthuysen, On the spin angular momentum of the Dirac particle, Nucl. Phys. 40 (1963) 1-12; J. Hilgevoord and E.A. De Kerf, The covariant definition of spin in relativistic quantum field theory, Physica 31 (1965) 1002-1016.

[16] Yu.N. Obukhov, A.J. Silenko, and O.V. Teryaev, Spin dynamics in gravitational fields of rotating bodies and the equivalence principle, Phys. Rev. D 80 (2009) 064044 (10 pages). 